\title{
Mapping the Most Massive Overdensities through Hydrogen (MAMMOTH). II. Discovery of the Extremely Massive Overdensity BOSS1441 at $z=2.32$
}

\author{
Zheng Cai ${ }^{1,2,9}$, Xiaohui Fan ${ }^{2}$, Fuyan Bian ${ }^{3}$, Ann Zabludoff ${ }^{2}$, Yujin Yang ${ }^{4}$, J. Xavier Prochaska ${ }^{1}$, Ian McGreer ${ }^{2}$, Zhen-Ya Zheng ${ }^{5,6}$, \\ Nobunari Kashikawa ${ }^{7}$, Ran Wang ${ }^{8}$, Brenda Frye ${ }^{2}$, Richard Green ${ }^{2}$, and Linhua Jiang ${ }^{8}$ \\ ${ }^{1}$ UCO/Lick Observatory, University of California, 1156 High Street, Santa Cruz, CA 95064, USA; zcai@ ucolick.org \\ ${ }^{2}$ Steward Observatory, University of Arizona, 933 North Cherry Avenue, Tucson, AZ 85721, USA \\ ${ }^{3}$ Research School of Astronomy \& Astrophysics, Mount Stromlo Observatory, Cotter Road, Weston ACT 2611, Australia \\ ${ }^{4}$ Korea Astronomy and Space Science Institute, 776 Daedeokdae-ro, Yuseong-gu Daejeon, Korea \\ ${ }^{5}$ Instituto de Astrofisica, Pontificia Universidad Catolica de Chile, 7820436 Santiago, Chile \\ ${ }^{6}$ Chinese Academy of Sciences South America Center for Astronomy, 7591245 Santiago, Chile \\ ${ }^{7}$ National Astronomical Observatory of Japan, Mitaka, Tokyo, 181-8588, Japan \\ ${ }^{8}$ The Kavli Institute for Astronomy and Astrophysics, Peking University, Beijing 100871, China \\ Received 2016 September 9; revised 2017 March 20; accepted 2017 March 27; published 2017 April 25
}

\begin{abstract}
Cosmological simulations suggest a strong correlation between high optical-depth Ly $\alpha$ absorbers, which arise from the intergalactic medium, and 3D mass overdensities on scales of 10-30 $\mathrm{h}^{-1}$ comoving Mpc. By examining the absorption spectra of $\sim 80,000$ QSO sight lines over a volume of $0.1 \mathrm{Gpc}^{3}$ in the Sloan Digital Sky Survey III (SDSS-III), we have identified an extreme overdensity, BOSS1441, which contains a rare group of strong Ly $\alpha$ absorbers at $z=2.32 \pm 0.02$. This absorber group is associated with six QSOs at the same redshift on a 30 comoving Mpc scale. Using Mayall/MOSAIC narrowband and broadband imaging, we detect Ly $\alpha$ emitters (LAEs) down to $0.7 \times L_{\mathrm{Ly} \alpha}^{*}$ and reveal a large-scale structure of LAEs in this field. Our follow-up LBT observations have spectroscopically confirmed 20 galaxies in the density peak. We show that BOSS1441 has an LAE overdensity of $10.8 \pm 2.6$ on a 15 comoving Mpc scale, which could collapse to a massive cluster with $M \gtrsim 10^{15} M_{\odot}$ at $z \sim 0$. This overdensity is among the most massive large-scale structures at $z \sim 2$ discovered to date.
\end{abstract}

Key words: galaxies: clusters: general - galaxies: high-redshift - intergalactic medium

\section{Introduction}

Understanding how cosmic fluctuations turn into structures and galaxies on large scales is one of the fundamental goals of observational cosmology. People have observed mature clusters at $z=1-2$ with a hot X-ray-emitting intracluster medium (ICM) and a mass of $\sim 10^{14} M_{\odot}$ (e.g., Gobat et al. 2011; Wang et al. 2016), indicating that the formation stage of these clusters and their progenitors (e.g., protoclusters) should be earlier than $z=2$ (e.g., Blakeslee et al. 2003; Fassbender et al. 2011). Determining the mass and abundance of the cluster progenitors at $z=2-3$ is crucial for building a complete picture of hierarchical growth in cosmic structure formation (e.g., Springel 2005; Chiang et al. 2013; Vogelsberger et al. 2014; Casey 2016).

Observations suggest that galaxy properties are closely related to the environment. The fundamental star formation-density and morphology-density relations are in place by $z=1$ (Postman et al. 2005; Smith et al. 2005) and do not strongly evolve from $z=1$ to 0 (De Lucia et al. 2012). Hierarchical galaxy formation models predict that these fundamental relations emerge at earlier epochs (e.g., $z=2-3$, Elbaz et al. 2007). Also, a large fraction of the present-day stellar mass was accumulated at $z \sim 2-3$ (e.g., Dickinson et al. 2003; Drory et al. 2005). Massive overdensities at $z=2-3$ are excellent laboratories to study the emergence of the environmental dependence of galaxy properties and the assembly of massive galaxies (e.g., Peter et al. 2007; Uchimoto et al. 2012). In addition, the overdense regions at $z=2-3$ are excellent sites to search for Ly $\alpha$ blobs (e.g., Prescott et al. 2009; Yang et al. 2009) and enormous Ly $\alpha$ nebulae (ELANe; e.g., Cantalupo et al. 2014;

\footnotetext{
${ }^{9}$ Hubble Fellow.
}

Hennawi et al. 2015; Cai et al. 2017). Utilizing these extended sources, the interactions between intergalactic/circumgalactic gas and massive galaxies can be revealed through the Ly $\alpha$ emission (e.g., Martin et al. 2015).

In the past few years, people have successfully discovered $\gtrsim 20$ galaxy overdensities at $z>2$. There are different approaches to tracing the overdense regions at high redshifts, such as with deep redshift surveys of galaxies (e.g., Steidel et al. 1998, 2005; Chiang et al. 2014; Lee et al. 2014) or with biased halos such as QSOs (e.g., $\mathrm{Hu}$ et al. 1996), high- $z$ submillimeter galaxies (Casey et al. 2015; Casey 2016), and radio galaxies (Venemans et al. 2007). Nevertheless, current galaxy redshift surveys are often limited by relatively small survey areas (up to a few $\mathrm{deg}^{2}$ ). Biased halos may have relatively small duty cycles (White et al. 2012), which make the overdensity selection to be incomplete. Due to these difficulties, a uniform sample at $z>2$ that contains a sufficient number of overdensities/protoclusters for robust comparisons between observations and hierarchical structure formation models (Chiang et al. 2013; Lee et al. 2014) has not yet been constructed. This observational challenge motivates us to devise a more complete technique of identifying galaxy overdensities at $z>2$, especially the most massive overdensities, which place the most stringent constraints on models of structure formation.

Cosmological simulations (e.g., Kollmeier et al. 2003; Cai et al. 2016) have demonstrated that the Ly $\alpha$ optical depth is closely correlated with mass over scales of $10-40 h^{-1}$ comoving Mpc (cMpc). Cai et al. (2016) demonstrate that the Ly $\alpha$ opacity increases with mass overdensities on $\approx 15 h^{-1} \mathrm{Mpc}$ scales, and overdensities traced by strong intergalactic medium (IGM) 
absorption are more massive than that traced by other rare sources (e.g., quasars). Guided by these simulations, Cai et al. (2016) have developed a novel approach for identifying extreme mass overdensities at $z=2-3$ on scales of $10-40 h^{-1} \mathrm{cMpc}$. This approach utilizes the largest library of quasar spectral library from the SDSS-III Baryon Oscillations Spectroscopic Survey (BOSS; e.g., Dawson et al. 2013). The BOSS quasar spectral library enables us to locate extremely rare, strong intergalactic $\mathrm{H}$ I (Ly $\alpha$ ) absorption systems and absorption groups. This approach also allows us to cover a significantly larger survey volume $\left(\sim \mathrm{Gpc}^{3}\right)$ and has less selection biases, because the $\mathrm{H}$ Idensity is correlated with the underlying dark matter density field over large scales (Cai et al. 2016).

In this paper, we present the first field (BOSS1441) selected from the early BOSS data release (DR9) using this novel technique. This field contains a group of high optical-depth Ly $\alpha$ absorption at $z=2.32 \pm 0.02$, and it is further associated with a QSO overdensity at $z=2.32 \pm 0.02$. This group of $\mathrm{Ly} \alpha$ absorption and QSOs traces an extremely massive overdensity of Ly $\alpha$ emitters (LAEs) at $z=2.32$. In the density peak (on $15 \mathrm{cMpc}$ scale), the LAE overdensity reaches $\approx 10.8$, making this field one of the most overdense high- $z$ structures discovered to date.

This paper is structured as follows. In Section 2, we introduce the SDSS-III/BOSS QSO spectral library and the target selection of the BOSS1441 overdense field. In Section 3, we introduce the observations in this field, including the deep narrowband, multiple-wavelength broadband imaging and the multiple-object spectroscopic follow-ups. In Section 4, we describe the observational results. We present the large-scale structure BOSS1441, and we quantify the overdensity using LAEs. Throughout this paper, we use the $A B$ magnitude system. When measuring distances, we refer to comoving separations or distances. We use cMpc to represent comoving $\mathrm{Mpc}$, and $\mathrm{pMpc}$ to represent physical Mpc. In this paper, the comoving distances do not contain the inverse $h$ factor unless we explicitly include it. We convert redshifts to comoving distances assuming a $\Lambda$ CDM cosmology with $\Omega_{m}=0.3$, $\Omega_{\Lambda}=0.7$, and $h=0.70$.

\section{Target Selection}

\subsection{SDSS-III/BOSS QSO Library}

We used the QSO spectral library observed in SDSS-III BOSS (Dawson et al. 2013; Ahn et al. 2014) to select fields that contain the group of high optical-depth, IGM Ly $\alpha$ absorption. BOSS is one of four surveys in SDSS-III, and it is a spectroscopic survey using the $2.5 \mathrm{~m}$ Sloan telescope (Gunn et al. 2006). SDSS-III spectra have a resolution of $R \approx 2000$, with spectral coverage from $3600 \AA$ to $10400 \AA$ (Bolton et al. 2012; Ross et al. 2012; Dawson et al. 2013). For each plate, the spectra have a typical exposure time of $1 \mathrm{hr}$, yielding a median signal-to-noise ratio $(\mathrm{S} / \mathrm{N})$ of $\sim 2$ per pixel at the QSOs' rest-frame wavelength $\lambda=1041-1185 \AA$ (Lee et al. 2012). A pixel in the spectra at $\lambda \approx 4000 \AA$ approximately covers $1 \AA$, which is equivalent to 1 comoving Mpc (cMpc) at $z \approx 2.3$. We selected the first field from the SDSS-III DR9 QSO catalog (Pâris et al. 2012). The SDSS-III DR9 QSO catalog contains $\sim 80,000$ QSOs over $3000 \mathrm{deg}^{2}$, yielding an average QSO density of 1 per $(15 \operatorname{arcmin})^{2}$, where (15 $\operatorname{arcmin})^{2}=\left(17 h^{-1} \mathrm{cMpc}\right)^{2}$ at $z \approx 2.3$. In order to identify absorption systems in the QSO spectra, we use a mean-fluxregulated principal component analysis (MF-PCA) technique to fit the QSO continuum fitting (Lee et al. 2013). The MF-PCA technique utilizes the PCA fitting to fit the shape of the Ly $\alpha$ forest continuum. The slope and amplitude of the continuum are adjusted using the external constraints of the mean optical depth of the Ly $\alpha$ forest (Lee et al. 2012; Becker et al. 2013).

\subsection{NB403 Narrowband Filter}

The narrowband filter $N B 403$ centered at $\lambda_{c}=4030 \AA$, with an FWHM of $\approx 40 \AA$ (Yang et al. 2009), corresponds to a distance of $\approx 40 \mathrm{cMpc}$ along the redshift direction. We constrain our selection within $z=2.32 \pm 0.02$ to match our custom narrowband filter $N B 403$. $N B 403$ is an ideal filter for this survey: the central wavelength corresponds to Ly $\alpha$ emission $z=2.32$, ideal for searching overdensities using the SDSS-III Ly $\alpha$ survey, because the QSO density peaks at slightly higher redshifts of $z \approx 2.3$.

\subsection{Selection of the BOSS1441 Field from SDSS-III/DR9}

We currently treat DLAs as contaminants of the IGM absorption systems. From cosmological simulations, IGM absorption systems effectively trace the most massive large-scale structures. Further, cosmological simulations predict a mass distribution of large-scale structures that can be tested in observations (Cai et al. 2016). DLAs can also yield strong Ly $\alpha$ absorption that resembles IGM absorption, but a large number of DLAs does not trace massive overdensities. Thus, we tried to rule out DLAs and effectively select absorptions due to IGM overdensities. Note IGM overdensities trace filamentary structures that could extend a few tens of Mpc, and thus a group of strong IGM absorption can be found in the large-scale structure. Cai et al. (2016) proposed the following four criteria that can be used to further rule out DLAs and effectively select overdensities traced by strong IGM absorption. The criteria include (a) $w_{0.8}<70 \AA$, where $w_{0.8}$ is defined as the width at flux/continuum $=0.8^{10}$ (b) The mean flux of the absorption trough $\left(F_{\text {trough }}\right)>0.15$, where the $F$ is the transmitted flux normalized using the continuum described in Section 2.1. ${ }^{11}$ (c) Non-detection of low-ionization metal lines associated with the Ly $\alpha$ absorption systems. ${ }^{12}$ (d) The presence of a group of absorption systems with $\geqslant 4$ absorption systems in a volume of $\left(20 h^{-1} \mathrm{cMpc}\right)^{3}$, where each absorption has a $\tau_{\text {eff }}^{15 h^{-1} \mathrm{cMpc}} \geqslant 3 \times$ mean optical depth $\left\langle\tau_{\text {eff }}\right\rangle \cdot{ }^{13}$ In this paper, our

\footnotetext{
${ }^{10}$ In Cai et al. (2016), we propose that the following (a)-(d2) criteria can be used to select IGM absorptions that trace massive overdensities. Here, we briefly summarize them. We use (a) to eliminate DLAs with column density $N_{\mathrm{HI}}>10^{20.6} \mathrm{~cm}^{-2}$. In the simulation (Figure 4 of Cai et al. 2016), even the most extreme IGM Ly $\alpha$ absorbers selected from a $1\left(h^{-1} \mathrm{Gpc}\right)^{3}$ volume have $w_{0.8}<70 \AA$. Wider absorbers are due to DLAs.

${ }^{11}$ We use criterion (b) to rule out DLAs using the flux in the dark trough. The absorption trough is defined as $\pm 5 \AA$ around the central pixel. The central pixel is defined as the pixel that gives the smallest transmitted flux over $15 \mathrm{~h}^{-1} \mathrm{Mpc}$. DLAs have the expected flux of zero in the dark trough; however, for IGM absorptions, the absorption trough still has transmitted flux larger than 0.1 in the simulation.

${ }^{12}$ For criterion (c), we use the low-ionization (low-ion) metal lines that are expected to trace high column-density H I clouds (e.g., Ford et al. 2013). However, if the quasar sight lines pass through the IGM overdensity, we expect no low-ion metal lines can be found in the BOSS spectra (e.g., Oppenheimer et al. 2012)

13 In cosmological simulations, using the BOSS sight line density, we found that a considerable fraction of IGM absorption is associated with other nearby IGM Ly $\alpha$ forest systems with $\tau_{\text {eff }}>0.8(3 \times\langle z\rangle)$. This effect arises because true IGM overdensities trace filamentary structures that could extend a few tens of comoving Mpc. However, DLAs or sub-DLAs, which are more likely to trace individual galaxies, normally have small H I cross-sections $(\sim 100$ physical kpc $\times 100$ physical kpc; e.g., Cai et al. 2014), and there is a small chance of finding a group of DLAs on Mpc scales.
} 


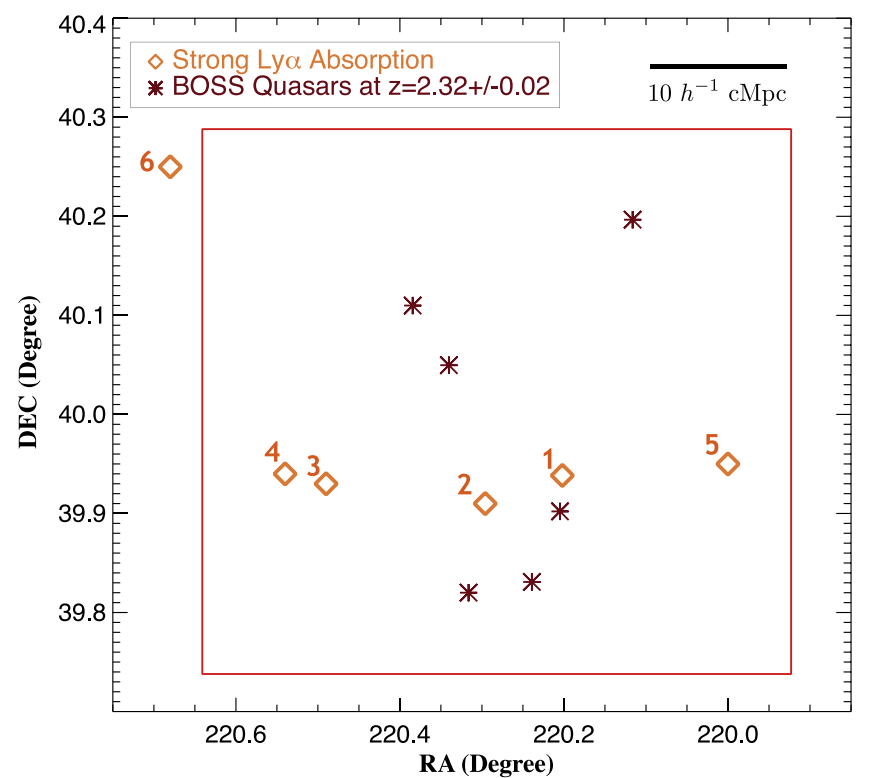

Figure 1. BOSS1441 field selected from the $3000 \mathrm{deg}^{2}$ SDSS-III/BOSS DR9 survey. This field contains a group of strong Ly $\alpha$ absorption systems (orange diamonds) and QSOs (brown asterisks) over $30 h^{-1}$ comoving Mpc at $z=2.3$. Each Ly $\alpha$ absorption is presented in Figures 2 and 3.
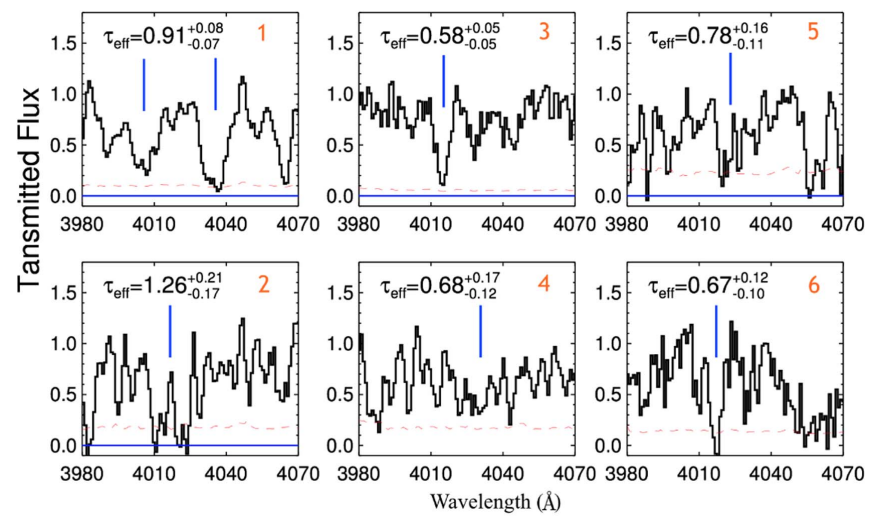

Figure 2. Snippets of the absorption group in the field of BOSS1441. We present the continuum-normalized QSO spectra at the Ly $\alpha$ absorption at $z=2.3$ in each panel. In each spectrum, we detect deep Ly $\alpha$ absorption (blue bar) at the same redshift as the QSO group. The blue bar represents the position that gives the lowest transmitted flux on the $15 h^{-1} \mathrm{Mpc}$ scale. The errors are presented using the red dashed line. The mean optical depth at $z=2.3$ is $\approx 0.19$ (e.g., Becker et al. 2013).

effective optical depth is calculated in the following way. We first smooth the spectrum over $15 h^{-1} \mathrm{Mpc}$ (20 pixels at $z=2.3$ ), and calculated the average flux on $15 h^{-1} \mathrm{Mpc}\left(\left\langle F_{15 h^{-1} \mathrm{Mpc}}\right\rangle\right)$. Then, the effective optical depth is calculated as follows: $\tau_{\mathrm{eff}}^{15 h^{-1} \mathrm{cMpc}}=$ $-\log \left(\left\langle F_{15 h^{-1} \mathrm{cMpc}}\right\rangle\right)$.

We automatically searched for $\operatorname{Ly} \alpha$ regions with an optical depth on a $15 h^{-1} \mathrm{cMpc}$ scale $\left(\tau_{\text {eff }}^{15 h^{-1} \mathrm{Mpc}}\right)$ greater than $3 \times\left\langle\tau_{\text {eff }}\right\rangle$ within $z=2.32 \pm 0.02$. We excluded strong Broad Line Absorptions (BALs) and Damped Ly $\alpha$ Absorption systems (DLAs) using our selection criteria (a)-(c). We also checked that these absorption systems are not flagged as DLAs in the SDSSIII/BOSS DLA catalog (Noterdaeme et al. 2012). We then checked the spectra of the nearby QSOs for absorption systems at a similar redshift, and identified fields containing $\geqslant 4$ absorption systems (in independent QSO sight lines) with $\tau_{\text {eff }}^{15 h^{-1} \mathrm{Mpc}}$ $\geqslant 3 \times\left\langle\tau_{\text {eff }}\right\rangle$ within $20 h^{-1} \mathrm{cMpc}$ scales. In total, we selected 11 fields that contain such absorption groups. In these 11 fields, we chose our first field with a central coordinate of $\alpha=14: 41: 28.80, \delta=+40: 01: 48$ (J2000). This field contains a group of six absorption systems (Figure 1), and the absorption group is further associated with six QSOs residing in the same field and at the similar redshift of $z=2.32 \pm 0.02$. In Figure 2, we show the Ly $\alpha$ absorption spectra of this group. We further present the ratio of the $\tau_{\text {eff }}^{15 h^{-1} \mathrm{Mpc}}$ to the mean optical depth $\left(\left\langle\tau_{\mathrm{eff}}\right\rangle\right)$ at $z=2.3$ (Figure 3). In both Figures 2 and 3, the two absorption systems (Absorption 1 and Absorption 2) on the left have the highest effective optical depth (with $\tau_{\text {eff }}^{15 h^{-1} \mathrm{cMpc}} \geqslant 4.5 \times\left\langle\tau_{\mathrm{eff}}\right\rangle$, the threshold of coherently strong $\operatorname{Ly} \alpha$ absorption proposed by Cai et al. 2016). BOSS1441 contains the most IGM absorption candidates and QSOs among all 11 fields. We then selected the BOSS1441 field to be the highest priority field in our sample. We adopted a mean optical depth $\left(\left\langle\tau_{\text {eff }}\right\rangle\right)$ to be 0.19 at $z=2.3$ (e.g., Becker et al. 2013). This absorption group, together with the QSO group, makes this field our highest priority candidate to search for the large-scale structure. We refer to this overdensity as BOSS1441.

\subsection{Survey Volume}

BOSS1441 is selected from SDSS-III/DR9. In the SDSSIII/DR9 database, we chose fields that can be observed in the spring semester from R.A. $=9 \mathrm{hr}$ to R.A. $=16 \mathrm{hr}$. We selected fields that contain $\geqslant 4$ background QSOs $\left(z_{\mathrm{em}}>2.34\right)$ within a $20 h^{-1} \mathrm{cMpc} \times 20 h^{-1} \mathrm{cMpc}$ area. We have 2622 independent fields that contain groups of background QSOs. The narrowband filter has an FWHM of $40 \AA$, corresponding to the radial separation of $42 \mathrm{cMpc}$. The survey volume of the 2622 groups is $2622 \times 20 h^{-1} \mathrm{cMpc} \times 20 h^{-1} \mathrm{cMpc} \times 42 \mathrm{cMpc} \approx 1.0 \times$ $10^{8} \mathrm{cMpc}^{3}$. Thus, BOSS1441 can be regarded as the most extreme system selected from a $\approx 1.0 \times 10^{8} \mathrm{cMpc}^{3}$ survey volume using our selection technique.

\section{Deep Imaging and Spectroscopy in the BOSS1441 Field}

After selecting the BOSS1441 field, we conducted deep narrowband and broadband imaging and multiple-slit spectroscopy to search for LAEs in this field.

\subsection{KPNO-4 m/MOSAIC Narrowband + Broadband Imaging}

We used the MOSAIC1.1 camera (Sawyer et al. 2010) at the f/3.1 prime focus of the $4 \mathrm{~m}$ Mayall Telescope at the Kitt Peak National Observatory to conduct deep narrowband and broadband imaging. We obtained deep narrowband images with the NB403 narrowband filter. As shown in Section 2.2, NB403 is used for probing the Ly $\alpha$-emitting galaxies at $z \approx 2.32$. The BOSS1441 overdensity was observed on 2013 March, and 2014 April and June. The total exposure time is $17.9 \mathrm{hr}$, which consists of individual 15 or 20 minute exposures with a standard dither pattern to fill in the gaps between the eight chips. The seeing ranges from 1 !" 1 to 1 !"7, with a median combined seeing of 1 !"32. We observed with the broadband filter $(\mathrm{Bw})$ for $3 \mathrm{hr}$ to match our selection criteria. These observing conditions enable us to achieve a narrowband magnitude $m_{N B 403}=25.1$ at $5 \sigma$ using a circle aperture of 2."5 and a $B w$-band magnitude $m_{B}=25.9$ at $5 \sigma$ using the same aperture. The narrowband sensitivity limit corresponds to a $1 \sigma$ Ly $\alpha$ surface brightness of $\mathrm{SB}_{\mathrm{Ly} \alpha}=2.4 \times 10^{-18} \mathrm{erg} \mathrm{s}^{-1} \mathrm{~cm}^{-2}$ $\operatorname{arcsec}^{-2}$. 

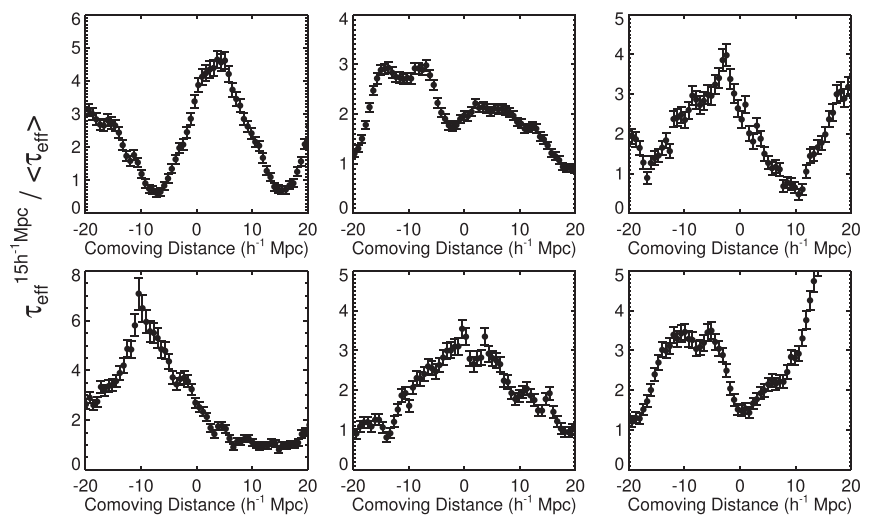

Figure 3. Effective optical depth over $15 h^{-1} \mathrm{cMpc}\left(\tau_{\mathrm{eff}}^{15 h^{-1} \mathrm{cMpc}}\right)$ in the BOSS1441 field. The zero point at the $x$-axis corresponds to $z=2.32$ for each QSO spectrum in Figure 2. In each spectrum, we see strong Ly $\alpha$ absorption with high optical depth. This group of $\operatorname{Ly} \alpha$ absorption traces the large-scale structure BOSS1441.

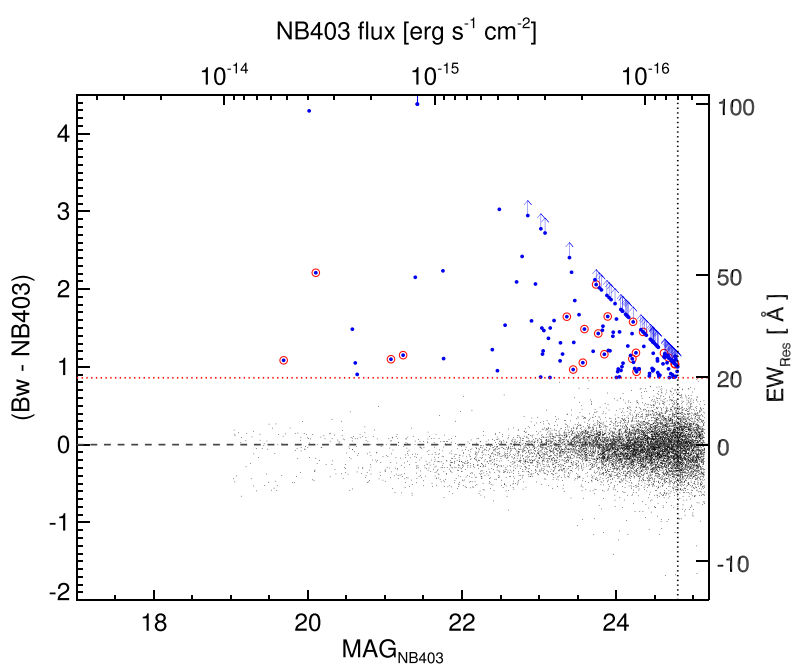

Figure 4. $(B w-N B 403)$ color-magnitude diagram for all sources detected in BOSS1441. The narrowband data reach $N B 403=24.8$ at the $5 \sigma$ level. We select line-emission objects (blue points) with the criteria $N B<24.3$ (vertical black line) and $B w-N B 403>0.87$ (red dashed line; equivalent to rest-frame EW > $20 \AA$ ). The red circles indicate LAEs that have been spectroscopically confirmed by our LBT/MODS observations.

\subsection{LBT Multiple-wavelength Broadband Imaging}

Our multiband imaging was conducted with the Large Binocular Cameras (LBC; Giallongo et al. 2008) at the Large Binocular Telescope (LBT) on Mount Graham in Arizona. LBC is characterized by a unique optical system. Each of the two mirrors has a collecting area of $8.4 \times 8.4 \mathrm{~m}^{2}$ and a field of view of $23^{\prime} \times 23^{\prime}$.

For a complete selection of star-forming galaxies at $z \approx 2.3$, we used the LBT to conduct deep multiple broadband observations in the $U, V$, and $i$ filters (Steidel et al. 2005). Multiple broadband imaging can also eliminate the low-redshift [O II] interlopers that are the main contaminants of LAEs at $z=2.3$. We use the standard $z=2$ star-forming galaxy selection (BX selection) technique (Adelberger et al. 2005) to eliminate the low- $z$ interlopers. The one-night LBT/LBC deep multicolor imaging was carried out in 2014 March. The LBC binocular mode was used, and the LBC blue channel was used solely for deep $U$-band observations. On the red side, we

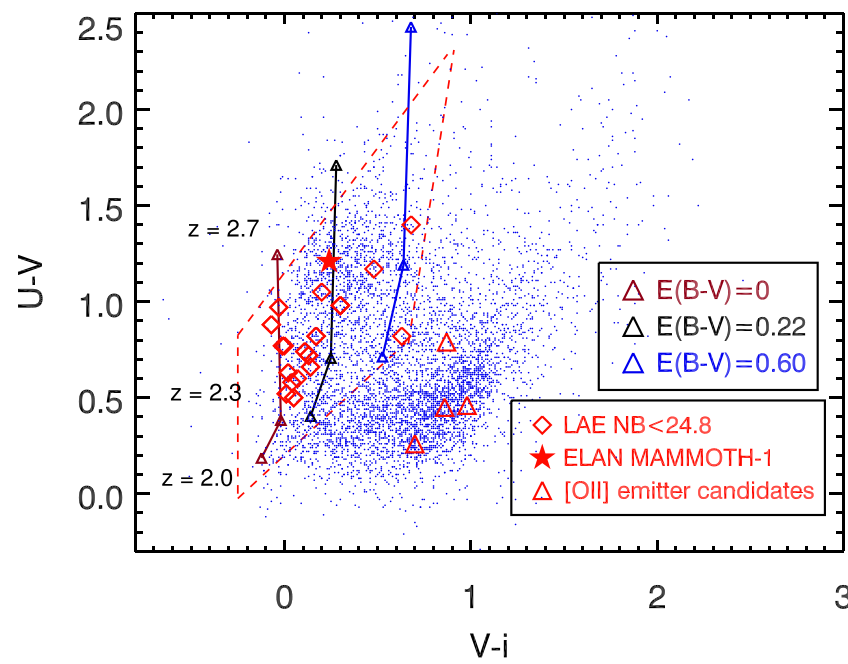

Figure 5. Broadband color of the Ly $\alpha$ emitters (LAEs) in the density-peak region of $15 \mathrm{cMpc}^{3}$. The broadband colors in $U, V$, and $i$ are obtained using the Large Binocular Camera (LBC) on the Large Binocular Telescope (LBT). The dotted red lines outline the selection region, and the photometric uncertainties is $\Delta z=0.15$ (Reddy et al. 2008). Most of the LAE candidates have broadband color consistent with the $z=2.0-2.7$ galaxies (BX galaxies). There are four emitters (red triangles) located at the low $z$ galaxy locus. These four galaxies are expected to be strong [O II] emitters with equivalent width $>60 \AA$ at $z=0.08 \pm 0.008$. All the other LAE candidates have broadband color consistent with high- $z$ LAEs.

observed the same field in the $V$ and $i$ filters. We conducted $6 \mathrm{hr}$ deep imaging in $U, 2.5 \mathrm{hr}$ in $V$, and $3.5 \mathrm{hr}$ in $i$. The individual exposure was six minutes with a standard five-point dither pattern to fill in the CCD gaps. The conditions were partially cloudy, with a median seeing of 0 ". 6 . Our observations reached $U_{\mathrm{AB}}=26.5$ at $5 \sigma, V_{\mathrm{AB}}=26.4$ at $5 \sigma$, and $i_{\mathrm{AB}}=26.4$ at $5 \sigma$. These depths are deep enough for selecting $0.5 \times L^{*}$ galaxies (e.g., Adelberger et al. 2003).

\subsection{LBT Multiple-slit Spectroscopy}

We used the Dual channel of the Medium-Dispersion Grating Spectrograph (MODS; Byard \& O'Brien 2000) to spectroscopically confirm of galaxies in the overdense field. LBT/ MODS has a field of view of $6^{\prime} \times 6^{\prime}$ for each blue and red channel, and it provides high efficiency in the full wavelength range of $3200-10000 \AA$ with $R=2000$ for most of the slits. We used a dichroic that divides the incoming beam at $\approx 5700 \AA$. This configuration covered Ly $\alpha$ and interstellar lines, such as $\mathrm{C}$ IV, $\mathrm{He}$ II, and C III], for LAEs at $z=2.3$.

On 2014 May 30 and 31, we conducted deep spectroscopy using two masks of LBT/MODS. Each mask contains 15 candidates of LAEs and BX galaxies. The first mask was anchored by an ELAN (see Section 4.1). We observed this mask for a total of six hours. The second mask was observed for over four hours. For each mask, we divided the total exposure into multiple individual $1800 \mathrm{~s}$ integrations. The average seeing for the MODS observations is $1^{\prime \prime}$. The MODS data reduction follows the LBT/MODS reduction routine. Each raw image was processed with the MODS CCD reduction utilities (modsTools v0.3) to obtain bias-subtracted and flat-fielded images. We fit the arc calibration with polynomials to determine the transformations between image pixels and wavelength. The sky model was fit to each image using B-splines and then subtracted. The LACOSMIC routine (van Dokkum 2010) was used to identify cosmic rays and to reject them during the construction of the sky model. The error 


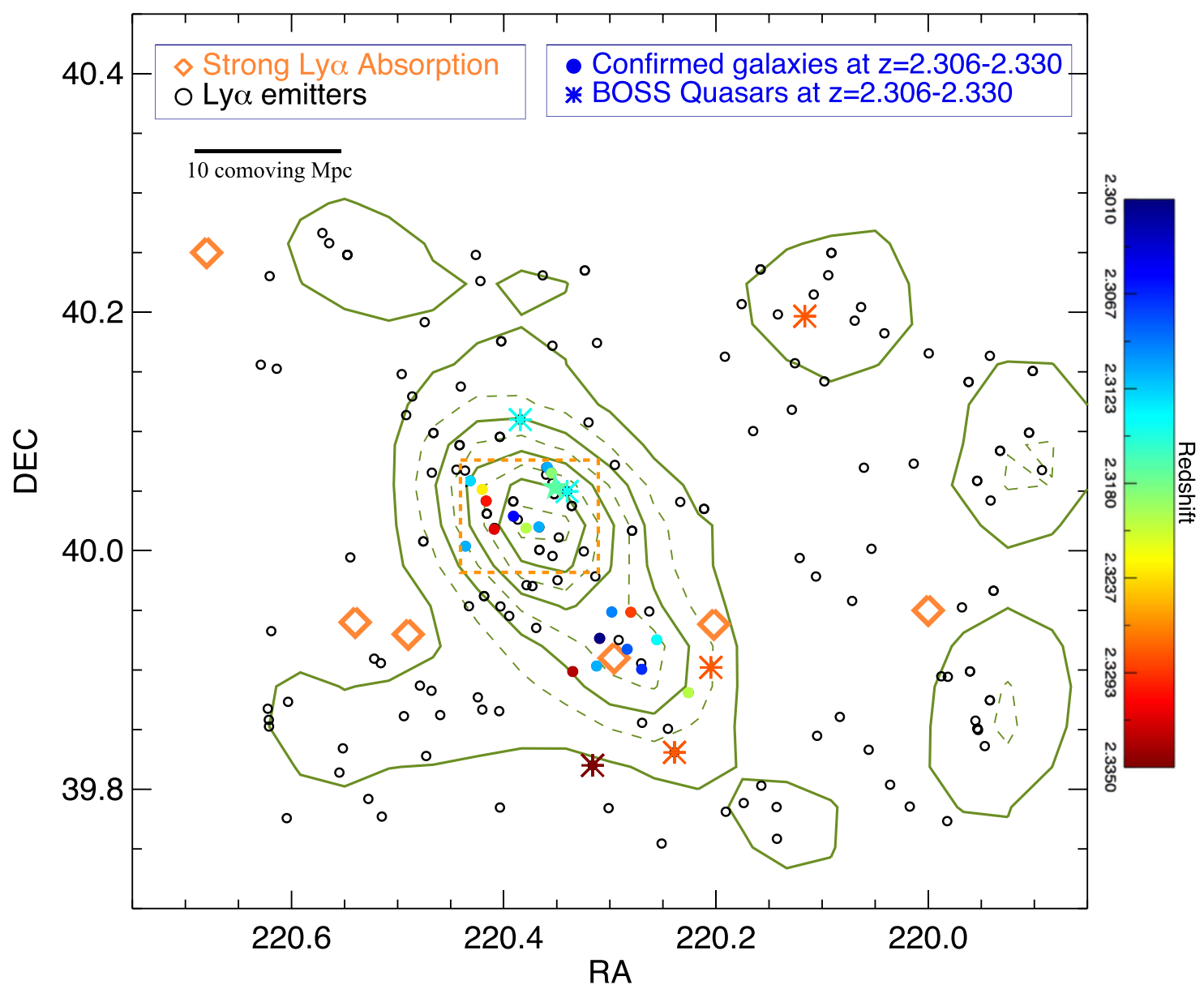

Figure 6. Galaxy overdensity BOSS1441 at $z=2.32 \pm 0.02$, selected from the SDSS-III/BOSS database. This structure is traced by a group of Ly $\alpha$ absorption systems (orange diamonds) and QSOs (brown asterisks) over $30 h^{-1} \mathrm{cMpc}$ at $z=2.3$. Each IGM absorption candidate is presented in Figures 2 and 3. Our LBT/ MODS spectroscopy targeted 20 LAEs with $\operatorname{mag}_{N B 403, \mathrm{AB}}<24.8$ with two masks in an area of $50 \operatorname{arcmin}^{2}\left(8 \mathrm{~h}^{-1} \times 8^{-1}\right) \mathrm{cMpc}^{2}$, and confirmed 19 of them. We colorcoded the spectroscopically confirmed galaxies and AGNs according to their redshifts. The LAE density in the density-peak region of $(15 \mathrm{cMpc})^{3}$ volume is $11.8 \times$ that in random fields $\left(\delta_{g}=10.8\right.$; red dashed box, $5.5 \mathrm{arcmin} \times 5.5 \mathrm{arcmin}$; also see footnote 14$)$. The green density contours are overplotted to show the density map of LAEs. Each contour (solid and dashed) indicates an increase in the galaxy number density of 0.1 galaxies per $\operatorname{arcmin}^{2}$, with the inner density peak of 1.0 per arcmin ${ }^{-2}$. We determine the redshift of each spectroscopically confirmed LAE based on the Ly $\alpha$ emission.

in the wavelength calibration due to the telescope flexure was corrected using sky lines.

\section{Results}

\subsection{Discovery of the Large-scale Structure BOSS1441}

Our narrowband imaging has a field of view of $33^{\prime} \times 33^{\prime}$ $\left(41 h^{-1} \times 41 h^{-1} \mathrm{cMpc}^{2}\right.$ at $\left.z=2.3\right)$ and a redshift range of $2.32 \pm 0.02$. In Figure 4, we present the color-selection criteria for the LAEs. We use the color criterion of $B w-N B>0.87$, corresponding to a Ly $\alpha$ rest-frame equivalent width of $\mathrm{EW}_{\mathrm{Ly} \alpha}>20 \AA$. We cut our selection of LAEs down to a narrowband magnitude of $m_{N B 403, \mathrm{AB}}=24.8$, where the LAE selection completeness is $\geqslant 90 \%$ (e.g., Guaita et al. 2011; Zheng et al. 2016). This limit corresponds to a Ly $\alpha$ luminosity of $1.56 \times 10^{42} \mathrm{erg} \mathrm{s}^{-1}, 0.73 L_{\mathrm{Ly} \alpha}^{*}$, where $L_{\mathrm{Ly} \alpha}^{*}=2.1 \times 10^{42}$ $\mathrm{erg} \mathrm{s}^{-1}$ (Ciardullo et al. 2012). The total volume of the MOSAIC imaging is $41 \times 41 \times 29.4 h^{-3} \mathrm{cMpc}^{3}$. Over this volume, we detect $99 \mathrm{LAE}$ candidates that satisfy the color criterion of $B w-N B>0.87$.

We use LBT/LBC to check the broadband colors of the LAE candidates. We use the $U-V$ versus $V-i$ colors of the LAE candidates. Following Adelberger et al. (2003), our selection criteria (red trapezoid in Figure 5) were found by running the
Bruzual \& Charlot (2003, hereafter BC03) models. We use a galaxy template with $0.2 \times Z_{\odot}$, constant star formation for $100 \mathrm{Myr}$, reddened by applying a Calzetti attenuation curve (Calzetti et al. 2000) with $E(B-V)=(0,0.22,0.6)$.

We find that BOSS1441 has a density peak at $\alpha=$ 14:41:26.40, $\delta=+40: 01: 12.0$. In that $6 \operatorname{arcmin} \times 6$ arcmin area, we detect 20 objects that satisfy the color criteria ${ }^{14}$ $B w-N B>0.87$ (red box in Figure 6); 15 have broadband colors in $U-V, V-i$ consistent with galaxies at $z \sim 2.3$. We present these LAE candidates in Figure 5 (red diamonds). Of the total of 21 sources including one QSO at $z=2.31$ (QSO3 as listed in Table 1), four are likely to be low-z [O II] emitters. Another one out of the 21 candidates have $B w>25.9$ and $i>26.5$. Therefore, we have 15 LAE candidates and one BOSS QSO in the density-peak region with $z \approx 2.3$. Among these LAEs, we detect an ELAN. We named this ELAN MAMMOTH-1 (blue star in Figure 6). The size of MAMMOTH-1 is extremely large, about $450 \mathrm{kpc}$ and among the largest ELANs discovered to date.

\footnotetext{
${ }^{14}$ We choose $5.5 \operatorname{arcmin} \times 5.5 \operatorname{arcmin}$ because we want to compare the volume in a $15 \mathrm{cMpc} \times 15 \mathrm{cMpc} \times 15 \mathrm{cMpc}$ box. 5.5 arcmin corresponds to $\approx 9 \mathrm{cMpc}$. The volume is $9 \mathrm{cMpc} \times 9 \mathrm{cMpc} \times 40 \mathrm{cMpc}$ (comoving distance along the sight-line direction $)=(15 \mathrm{cMpc})^{3}$, which has the same volume as the overdensity calculations in other fields (e.g., Chiang et al. 2014).
} 
Table 1

BOSS QSOs in BOSS1441

\begin{tabular}{lccc}
\hline \hline Name & R.A. & Decl. & Redshift \\
\hline BG QSO1 $^{\mathrm{a}}$ & $14: 40: 48.56$ & $+39: 56: 18.3$ & 2.542 \\
\hline BG QSO2 & $14: 39: 58.32$ & $+40: 03: 14.0$ & 2.422 \\
\hline BG QSO3 & $14: 41: 10.98$ & $+39: 54: 23.9$ & 2.999 \\
\hline BG QSO4 & $14: 42: 51.83$ & $+40: 14: 53.4$ & 2.553 \\
\hline BG QSO5 & $14: 42: 10.59$ & $+39: 56: 31.8$ & 2.612 \\
\hline BG QSO6 & $14: 39: 58.44$ & $+40: 03: 14.0$ & 2.425 \\
\hline QSO1 & $14: 40: 27.91$ & $+40: 11: 47.8$ & 2.324 \\
\hline QSO2 & $14: 40: 49.15$ & $+39: 54: 07.6$ & 2.323 \\
\hline QSO3 $^{\text {b }}$ & $14: 41: 21.67$ & $+40: 02: 58.9$ & 2.311 \\
\hline QSO4 & $14: 40: 57.39$ & $+39: 49: 51.2$ & 2.324 \\
\hline QSO5 $^{\text {QSO6 }}$ & $14: 41: 15.91$ & $+39: 49: 12.0$ & 2.334 \\
\hline
\end{tabular}

Notes.

a The background QSOs in this field (Figures 1,2).

${ }^{b}$ This QSO resides in the $(15 \mathrm{cMpc})^{3}$ density-peak region of BOSS1441.

MAMMOTH-1 also contains the strongly extended He II $\lambda 1640$ and C IV $\lambda 1549$ emission. We will discuss the MAMMOTH-1 nebula in our subsequent paper (Cai et al. 2017).

Our color criteria have a high successful rate for selecting galaxies at $z \approx 2.3$. In our LBT/MODS field, which completely covers the density-peak area $\left(\sim 50 \operatorname{arcsec}^{2}\right)$, we put 20 sources in the MODS mask that satisfy both the LAE and BX galaxy color-selection criteria. We spectroscopically confirm the Ly $\alpha$ emission for 19 of them with $m_{\mathrm{NB}}<24.8$. Eleven of the nineteen LAEs reside in the density-peak area (red box in Figure 6). Including the BOSS QSO in this region, we have $\operatorname{Ly} \alpha$-based redshifts for 20 galaxies in our MODS footprint and 12 galaxies in the density-peak region. We summarize the spectroscopically confirmed galaxies in Table 2 and present their Lyoemission lines in Figure 7. We did not detect Lyaemission for only one galaxy candidate, because the expected Ly $\alpha$ line falls in the gap between two CCDs of LBT/ MODS. The spectroscopic results demonstrate that our LAE selection is solid.

The main low $-z$ interlopers are strong [O II] emitters at $z=0.081 \pm 0.005$ with $\mathrm{EW}_{[\mathrm{O} \text { II] }}>60 \AA$. We excluded them from our spectroscopic follow-ups, because their broadband colors do not satisfy the high- $z$ LAE criteria. Our spectroscopic survey has a resolution of $R=2000$ and is able to resolve the $[\mathrm{O} \mathrm{II}] \lambda \lambda 3727,3729$ doublets. Our optical range covers the [O $\mathrm{III}], \mathrm{H} \beta$, and $\mathrm{H} \alpha$ lines of the emitters. Throughout the spectroscopic check, we did not find any $\mathrm{O}$ II interlopers that contaminate our LAE spectroscopic sample.

\subsection{LAE Overdensity in the Density Peak of BOSS1441}

Stark et al. (2015) find that the characteristic size of protoclusters (i.e., defined as progenitors of massive $z=0$ clusters) is about $15 \mathrm{cMpc}\left(\sim 10 h^{-1} \mathrm{cMpc}\right)$. Steidel et al. (1998, 2005) and Chiang et al. (2013) estimate the relation between the protocluster mass $m$ and galaxy overdensity $\delta_{g}$ in a volume of $(15 \mathrm{cMpc})^{3}$, where $\delta_{g}$ is defined as $\delta_{g}=\frac{N_{\text {group }}}{N_{\text {field }}}-1$, and $N_{\text {field }}$ is the mean number of LAEs in random fields. $N_{\text {group }}$ is the LAE number within the overdensity. For comparison to previous confirmed overdensities (e.g., see Table 4), let us first measure the galaxy overdensity in the density peak of an asymmetric box with a total volume of $15^{3} \mathrm{cMpc}^{3}$.

In Figure 8, we present the radial density profile (black dots with error bars) measured in circular annuli centered on the field center of BOSS1441 $(\alpha=14: 41: 26.40, \delta=+40: 01: 12.0)$. The $x$-axis is the diameter of the circle. The average overdensity of this protocluster is $>10$ over a large scale of $\gtrsim 15 \mathrm{cMpc}$. From our LBT/MODS spectroscopy, the LAEs in this region have $z=2.319 \pm 0.013$. According to the previous section, in the density peak of a $(15 \mathrm{cMpc})^{3}$ volume (red box), we detected 16 LAEs (including a BOSS QSO) down to $L_{\mathrm{Ly} \alpha} \geqslant 0.73 \times L_{\mathrm{Ly} \alpha}^{*}$. Twelve out of these sixteen LAE candidates are spectroscopically confirmed (including a BOSS QSO). According to the previous section, our LAE selection is solid. Assuming the other LAE candidates that we did not put in our mask are real, and we further assume a 90\% LAE completeness (Guaita et al. 2011; Zheng et al. 2016), the intrinsic LAE number should be 17.8 in the density peak. From the luminosity function of LAEs at $z=2.1$ (Ciardullo et al. 2012), the average LAE number is $N_{\text {field }}=1.53$ in a cube with $15 \mathrm{cMpc}$ on a side. We use the following equation to calculate the mean LAE density in random fields:

$$
N_{\text {field }}=\int_{0}^{(15 \mathrm{Mpc})^{3}} \int_{0.73 \times L^{*}}^{+\infty} \phi^{*}\left(L / L^{*}\right)^{\alpha} e^{-L / L^{*}} d\left(L / L^{*}\right) d V,
$$

where $\phi^{*}=10^{-2.86 \pm 0.05} \mathrm{Mpc}^{-3}, \alpha=-1.65$, and $\log \frac{L^{*}}{\left(\operatorname{erg~s}^{-1}\right)}=$ $42.33 \pm 0.12$. Then, we calculate that the LAE overdensity has $\delta_{g} \approx 10.8$, making this field one of the most overdense structures of LAEs ever discovered. In Table 4, we compare the overdensity of BOSS1441 with other spectroscopically confirmed, most massive large-scale structures. We will discuss this comparison in detail in the next section, Section 5.2.

Let us measure the uncertainties of the galaxy number counts between different $(15 \mathrm{cMpc})^{3}$ cells. Similar to Lee et al. (2014), we account for both Poissonian shot noise and sample variance. The total fractional error of $\frac{N_{\text {group }}}{N_{\text {field }}}$ is a quadratic sum of these three error sources. (1) Shot noise $\delta N_{\text {shot }}$. Using the Ly $\alpha$ luminosity function (Ciardullo et al. 2012), in random fields, $N_{\text {field }}$ is 1.53 within a $(15 \mathrm{cMpc})^{3}$ box. Thus, the fractional error from shot noise is $\delta N_{\text {shot }}=\sqrt{17.8}=4.22$, and $\frac{\delta N_{\text {shot }}}{N_{\text {field }}}=4.22 / 1.53=$ 2.76. (2) Sample variance $\left(\delta N_{\mathrm{SV}}\right) . \delta N_{\mathrm{SV}}$ is determined by putting random boxes in different positions with $(15 \mathrm{cMpc})^{3}$, and measuring the variance of the halo counts. The sample variance is measured to be $\frac{\delta N_{\mathrm{SV}}}{N_{\text {field }}}=52 \%$ (Chiang et al. 2013; Lee et al. 2014). (3) The uncertainties associated with the measurement of the luminosity function $\left(\delta N_{\mathrm{LF}}\right)$. The average galaxy number density has an uncertainty of $\delta N_{\mathrm{LF}} / N_{\text {field }}=5 \%$ (e.g., Ciardullo et al. 2012). Therefore, we measure the total fraction error of $\frac{\delta N_{\text {group }}}{N_{\text {field }}}=$ $\sqrt{\left(\frac{\delta N_{\text {shot }}}{N_{\text {field }}}\right)^{2}+\left(\frac{\delta N_{\mathrm{SV}}}{N_{\text {field }}}\right)^{2}+\left(\frac{\delta N_{\mathrm{LF}}}{N_{\text {field }}}\right)^{2}}=\sqrt{2.76^{2}+0.53^{2}+0.05^{2}} \approx$ 2.8 , which means that the root-mean-square (rms) fluctuation of the LAE number per $(15 \mathrm{cMpc})^{3}$ cell is roughly $1.5 \times 2.8=4.2$, i.e., the measured error of the LAE number per $(15 \mathrm{cMpc})^{3}$ cell is 4.2. Therefore, the galaxy overdensity $\left(\delta_{g}\right)$ in the density peak is $\delta_{g}=10.8 \pm 2.8$. One can further provide a more conservative 
Table 2

Nineteen Spectroscopically Confirmed Galaxies at $z=2.32 \pm 0.02$ in Our MODS Follow-up Observations (50 arcmin $)^{2}$

\begin{tabular}{|c|c|c|c|c|}
\hline Name & R.A. & Decl. & $L_{\mathrm{Ly} \alpha}\left(\mathrm{erg} \mathrm{s}^{-1}\right)$ & Redshift \\
\hline LAE1 & $14: 41: 44.67$ & $+40: 00: 13.52$ & $3.75 \pm 0.2 \times 10^{42}$ & 2.312 \\
\hline LAE2 & $14: 41: 43.58$ & $+40: 03: 31.15$ & $8.40 \pm 0.2 \times 10^{42}$ & 2.313 \\
\hline LAE3 & $14: 41: 40.86$ & $+40: 03: 05.21$ & $4.60 \pm 0.2 \times 10^{42}$ & 2.323 \\
\hline LAE4 & $14: 41: 39.97$ & $+40: 02: 30.07$ & $2.38 \pm 0.2 \times 10^{42}$ & 2.330 \\
\hline LAE5 & $14: 41: 38.04$ & $+40: 01: 08.39$ & $15.1 \pm 0.2 \times 10^{42}$ & 2.338 \\
\hline LAE6 & $14: 41: 31.01$ & $+40: 01: 09.72$ & $2.34 \pm 0.2 \times 10^{42}$ & 2.321 \\
\hline LAE7 & $14: 41: 27.98$ & $+40: 01: 10.79$ & $3.53 \pm 0.2 \times 10^{42}$ & 2.312 \\
\hline LAE8 & $14: 41: 26.22$ & $+40: 04: 12.02$ & $3.35 \pm 0.2 \times 10^{42}$ & 2.312 \\
\hline LAE9 & $14: 41: 25.30$ & $+40: 03: 55.89$ & $8.9 \pm 0.2 \times 10^{42}$ & 2.319 \\
\hline LAE10 (MAMMOTH-1 Nebula) & $14: 41: 24.31$ & $+40: 03: 11.91$ & $528 \pm 0.3 \times 10^{42}$ & 2.319 \\
\hline LAE11 & $14: 41: 33.82$ & $+40: 01: 42.75$ & $3.62 \pm 0.3 \times 10^{43}$ & 2.311 \\
\hline LAE12 & $14: 41: 20.39$ & $+39: 53: 55.39$ & $14.4 \pm 0.3 \times 10^{43}$ & 2.336 \\
\hline LAE13 & $14: 41: 15.03$ & $+39: 54: 12.10$ & $7.3 \pm 0.3 \times 10^{42}$ & 2.307 \\
\hline LAE14 & $14: 41: 14.15$ & $+39: 55: 35.57$ & $3.9 \pm 0.3 \times 10^{42}$ & 2.334 \\
\hline LAE 15 (AGN) & $14: 41: 11.72$ & $+39: 56: 54.96$ & $234.6 \pm 0.7 \times 10^{42}$ & 2.311 \\
\hline LAE16 & 14:41:08.10 & $+39: 55: 02.62$ & $3.2 \pm 0.3 \times 10^{42}$ & 2.309 \\
\hline LAE17 & $14: 41: 04.83$ & $+39: 54: 02.31$ & $3.5 \pm 0.3 \times 10^{42}$ & 2.326 \\
\hline LAE18 & $14: 41: 01.51$ & $+39: 55: 31.41$ & $4.2 \pm 0.3 \times 10^{42}$ & 2.317 \\
\hline LAE19 & $14: 40: 54.20$ & $+39: 52: 51.62$ & $1.7 \times 0.3 \times 10^{42}$ & 2.308 \\
\hline
\end{tabular}

estimate of the overdensity based merely on the mean density of LAEs in this field. Note that BOSS1441 is associated with a largescale structure, so that this method provides a conservative measurement and a lower limit of the overdensity. In the whole field, we have $99 \mathrm{LAE}$ candidates. In the central $6^{\prime} \times 6^{\prime}$ region, we have 19 LAEs. Excluding this region, we have 80 LAEs in the area except in the density peak, and the average density of LAEs in BOSS1441 is $0.08 \operatorname{arcmin}^{-2}$. In the density peak, the LAE density is $0.53 \mathrm{arcmin}^{-2}$. The overdensity of the density peak is 0.53 / $0.08-1=5.6$. This should be the lower limit of the overdensity.

\section{Discussion}

\subsection{Present-day Mass of BOSS1441}

We summarize the properties of BOSS1441 in Table 3. If BOSS1441 collapses to a cluster at $z \sim 0$, then the collapsed cluster mass can be estimated from the overdensity of galaxies at $z=2-3$ (e.g., Chiang et al. 2013). We measure the mass based on the estimation by Steidel et al. $(1998,2005)$ and Chiang et al. (2013). The progenitors of massive $z=0$ clusters have a characteristic size of $15 \mathrm{cMpc}$ (e.g., Stark et al. 2015). As shown in the previous section, BOSS1441 has an overdensity of $\delta_{g}=10.8$ over a $15 \mathrm{cMpc}$ scale. If the matter on a $15 \mathrm{cMpc}$ scale collapses to a bound, virialized system at $z \sim 0$, the virialized mass $\left(M_{z \sim 0}\right)$ can be expressed as

$$
M_{z=0}=\left(1+\delta_{m}\right)\langle\rho\rangle V_{\text {true }}=2.6 \times 10^{14}\left(1+\delta_{m}\right) / C h^{-1} M_{\odot},
$$

where $V_{\text {true }}$ is the true volume after correcting for redshift-space distortion. $V_{\text {true }}=V_{\text {app }} / C$ and $V_{\text {app }}=15^{3} \mathrm{cMpc}^{3}$, the observed comoving volume in which the measurement is made (within the $6^{\prime} \times 6^{\prime}$ region on the plane of sky and neglecting the peculiar velocities between $z=2.31-2.33$ ). $C$ is a volume factor correcting for the redshift-space distortion caused by peculiar velocities and defined as $\frac{V_{\text {app }}}{V_{\text {true }}} .\langle\rho\rangle$ is the mean matter density of the universe, which is equal to $\frac{3 H_{0}^{2}}{8 \pi G} \Omega_{m}=8.4 \times 10^{10} h^{2} M_{\odot} \mathrm{cMpc}^{-3}$. The $\delta_{m}$ is the matter overdensity. The relation between the observed galaxy overdensity and matter overdensity can be expressed as $1+b \delta_{m}=C\left(1+\delta_{\mathrm{g}}\right)$, where $\delta_{\mathrm{gal}, \text { obs }}$ is the observed galaxy overdensity we measured in Section 4.2 and the galaxy bias $b$ could range from 1 to 4 (e.g., Steidel et al. 1998). Dey et al. (2016) found that LAEs are a biased tracer of the matter distributions in an overdensity. They observe that Ly $\alpha$ emission is clearly enhanced in denser regions in their protocluster. We take the LAE bias to be $b_{\mathrm{LAE}}=2.1$ (e.g., Gawiser et al. 2007; Lee et al. 2014). The volume correction factor $C$ can be approximated by the expression $C=1+f-f\left(1+\delta_{m}\right)^{1 / 3}$ (Steidel et al. 1998, 2005), where $f=\Omega_{m}(z)^{0.6}$. For $\delta_{g}=10.8$ at $z=2.32$, we find $C=0.56$ and $\delta_{m}=2.2$. According to the theory of density perturbation, the overdensity $\delta_{m}=2.2$ corresponds to a linear overdensity $\delta_{L} \sim 0.83$ at $z=2.3$ (Equation (18) of Mo \& White). 

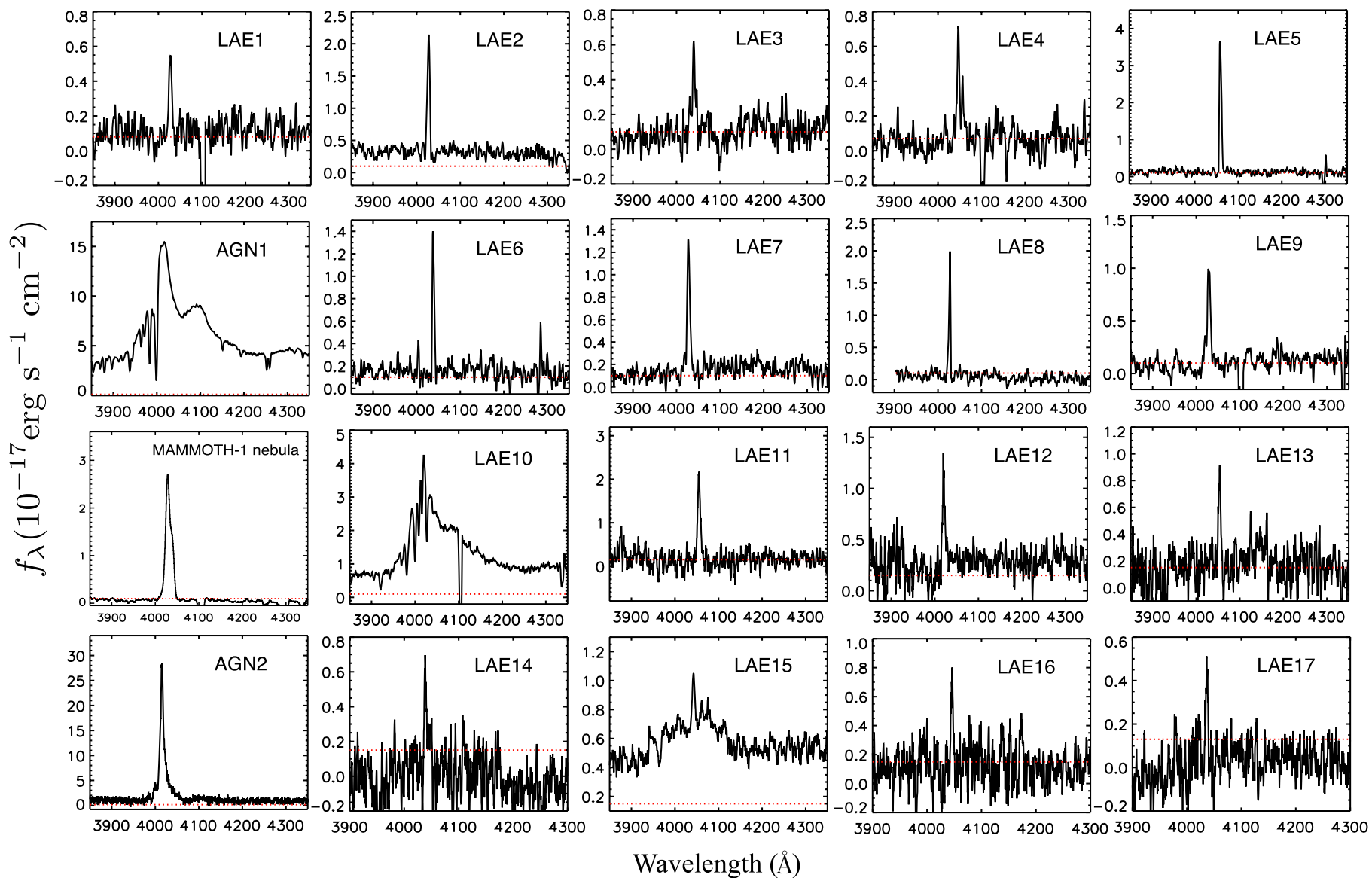

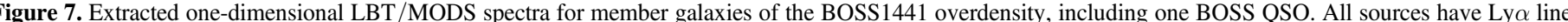
detections with $\mathrm{S} / \mathrm{N}>10$. The red dotted line indicates the statistical error, without considering the systematic uncertainties of sky subtraction.

Table 3

Galaxy Overdensity of BOSS1441

\begin{tabular}{lccccc}
\hline \hline $\begin{array}{l}\text { Volume } \\
(\mathrm{cMpc})^{3}\end{array}$ & $\begin{array}{c}\text { Depth } \\
L_{\mathrm{Ly} \alpha}^{*}\end{array}$ & $\begin{array}{c}\text { Random Fields }^{\mathrm{a}} \\
\text { LAE Number }^{\mathrm{b}}\end{array}$ & $\begin{array}{c}\text { MAMMOTH-1 } \\
\text { LAE Number }\end{array}$ & $\begin{array}{c}\text { Noise } \\
\text { Number }\end{array}$ & $\begin{array}{c}\text { Over- } \\
\text { density }\end{array}$ \\
\hline $15^{3}$ & 0.73 & 1.52 & 21 & 1.23 & $10.8 \pm 2.8$ \\
\hline
\end{tabular}

Notes.

a Number calculated from the Ly $\alpha$ luminosity function (Ciardullo et al. 2012) at $z=2.1$.

${ }^{b}$ Number of LAEs.

Following the growth of linear perturbation $\left(\delta_{L} \propto t^{2 / 3}\right), \delta_{L}$ should pass the threshold of $\delta_{L}>1.69$ at $z<0.5 . \delta_{L}>1.69$ corresponds to the density contrast of the gravitational collapse of a spherical perturbation (e.g., Jenkins et al. 2001; Reed et al. 2003). Thus, we expect that BOSS1441 should collapse to form a cluster at low redshift. Using cosmological simulations, Chiang et al. (2013) find that $\geqslant 80 \%$ of the overdensities with $\delta_{g}>2.88$ at $z \approx 2$ are protoclusters that will collapse to virialized clusters at $z \sim 0$.

Adopting an LAE bias of $b=2.1$ and a correction factor $C=0.56$, we estimate that BOSS1441 at $z=2.3$ should evolve into a $z=0$ cluster with a mass of $M_{z \sim 0} \approx 1.5 \times 10^{15}$ $M_{\odot}$. If we use the overdensity measured based on the mean LAE density in this field, the overdensity in the $(15 \mathrm{Mpc})^{3}$ volume is $\delta_{g}=5.6$ (see the conservative measurement at the end of Section 4.2), then the mass is also about $8.0 \times 10^{14} M_{\odot}$. Such a high mass makes this overdensity one of the most massive cluster progenitors (see Table 4). In addition to the analytical estimations, Chiang et al. (2013) calculated the collapsed cluster masses using cosmological simulation. Using the $\delta_{g}-\delta_{m}$ relation (Figure 7 of Chiang et al. 2013), we estimate that the most massive $M_{z=0} \geqslant 10^{15} M_{\odot}$ cluster has a progenitor at $z \approx 2.5$ with $\delta_{g} \approx 5-10$ and $\delta_{m} \approx 2-4$. In Figure 8 , we further compare the radial density profile of BOSS1441 (black points) with the predicted radial density profiles of cluster progenitors (Chiang et al. 2013). We find that the radial profile of BOSS1441 matches that of Coma cluster progenitors on scales $\sim 5-20 \mathrm{cMpc}$. On smaller scales of $<5 \mathrm{cMpc}$, the 


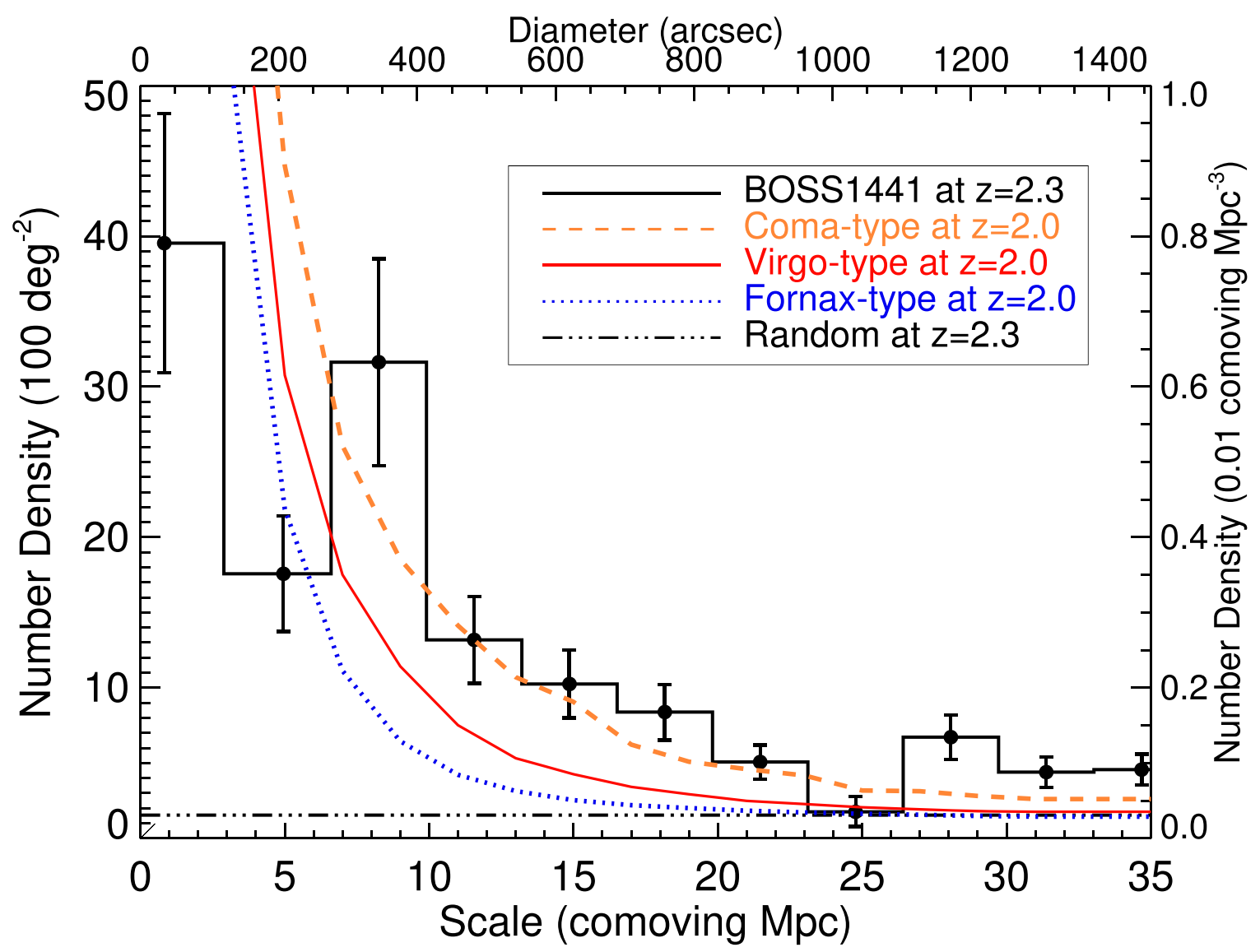

Figure 8. Radial density profile measured in circular annuli centered on the density peak of BOSS1441 $(\alpha=14: 41: 26.40, \delta=+40: 01: 12.0)$. The dotted horizontal line represents the number density at $z=2.3$ in random fields, calculated using the galaxy luminosity function in Ciardullo et al. (2012). This protocluster has one of the highest densities of $\operatorname{Ly} \alpha$ emitters found at $z=2-3$. We overplot the predicted radial density profile of different cluster progenitors at $z=2$ from cosmological simulations (Chiang et al. 2013). The orange dashed line represents the progenitors of Coma-like clusters $\left(M_{z=0}>10^{15} M_{\odot}\right)$, the red solid line represents the progenitors of Virgo-like clusters $\left(M_{z=0} \approx(3-10) \times 10^{14} M_{\odot}\right)$, and blue dotted line represents the progenitors of Fornax-like clusters $\left(M_{z=0} \approx(1-3) \times 10^{14} M_{\odot}\right)$.

Table 4

Comparisons of Protoclusters Traced by Different Techniques at $z \sim 2-3$

\begin{tabular}{|c|c|c|c|c|c|}
\hline Name & $\langle z\rangle$ & Scale & $\overline{\delta g}$ & Mass & $\overline{\text { Reference }}$ \\
\hline & & $(\mathrm{cMpc})$ & & $\left(10^{14} M_{\odot}\right)$ & \\
\hline CDFSz28 & 2.82 & 15 & {$[4.7,4.7,6.0,6.6]$} & {$[3.3,3.3,3.7,3.8]$} & 9 \\
\hline $\operatorname{cosmos} 34^{\mathrm{a}}$ & 3.04 & 15 & $2.28_{-0.79}^{+1.04}$ & $\cdots$ & 8 \\
\hline HS1700+643 & 2.300 & 19.5 & 5.7 (6.9 in paper) & 12 & 2,3 \\
\hline $\mathrm{J} 1431+3239$ & 3.733 & 15 & $4.2 \pm 0.9$ & 7 & 5 \\
\hline MRC 0943-242 & 2.92 & 20 & $2.2_{-0.7}^{+0.9}$ & $4-5$ & 4,5 \\
\hline SSA22 & 3.09 & 23 & $3.6_{-1.2}^{+1.4}$ & $\sim 10$ & 1 \\
\hline TNJ1338-1942 & 4.1 & 18 & $3.8_{-0.8}^{+1.1}$ & 7 & $4,5,6$ \\
\hline This work & $2.329 \pm 0.013$ & 15 & $10.8 \pm 2.8$ & 10 & \\
\hline This work & $2.329 \pm 0.013$ & 19.5 & $8.3 \pm 2.0$ & 20 & \\
\hline
\end{tabular}

Notes. Summary of massive overdensities discovered at $z \sim 2-3$ with mass $>5 \times 10^{14} M_{\odot}$. The mass is determined consistently by setting galaxy bias of Ly $\alpha$ emitter to be $b_{\mathrm{LAE}}=2.1$.

${ }^{a}$ COSMOS34 has the highest $\delta_{g}$ in all protoclusters in the cosmic field (Chiang et al. 2013).

b TNJ1338-1942 is the most massive protocluster discovered using radio galaxies as a tracer (Venemans et al. 2007).

References. (1) Steidel et al. (1998), (2) Steidel et al. (2005), (3) Digby-North et al. (2010), (4) Venemans et al. (2007), (5) Venemans et al. (2005), (6) Venemans et al. (2002), (7) Lee et al. (2014), (8) Chiang et al. (2014), (9) Zheng et al. (2016).

observed galaxy number density is significantly lower than that of Coma cluster progenitors in cosmological simulations. This discrepancy may indicate that the galaxies in the density peak are not completely traced by LAEs (e.g., Muldrew et al. 2015). In the future, we need to conduct a complete galaxy survey from the optical to the infrared, and study whether the LAE is a 
good tracer in the density peak, and whether quenched galaxies exist in the density peak (Feldmann \& Mayer 2015).

In Cai et al. (2016), we showed the $\delta_{m}-\tau$ relation from cosmological simulations. The BOSS1441 overdensity is our first observed field that is traced by $\operatorname{Ly} \alpha$ absorbers. It is interesting to check if BOSS1441 follows the $\delta_{m}-\tau$ relation in the simulation. In the LyMAS simulation (Cai et al. 2016), we found that the mass overdensities can be traced by groups of strong Ly $\alpha$ absorption systems. The absorption group is defined as $\geqslant 4$ absorptions with $\tau_{\text {eff }}^{15 h^{-1} \mathrm{cMpc}}>3 \times\left\langle\tau_{\text {eff }}\right\rangle$ on 20 $h^{-1} \mathrm{cMpc}$, and each group contains at least one strong IGM absorption candidate with $\tau_{\text {eff }}^{15 h^{-1} \mathrm{cMpc}} \geqslant 4.5 \times\left\langle\tau_{\text {eff }}\right\rangle$. Such absorption groups trace overdensities within the range of $\delta_{m}=0.6-2.8$, with a median value of 1.50 . From our LAE observations, BOSS1441 $\left(\delta_{m}=2.2\right)$ is within this range.

\subsection{Rarity of this Structure}

As shown in Section 5.1, BOSS1441 could collapse to a bound, virialized system at $z=0$ with a virialized mass $\left(M_{z=0} \approx 10^{15} M_{\odot}\right)$. Bahcall \& Cen (1993) present the cluster mass function at $z \lesssim 0.2$. Extrapolating this cluster mass function to the high-mass end (Bahcall et al. 2003; Casey 2016), we estimate that the volume density of high-mass clusters $\left(M>10^{15} M_{\odot}\right)$ is $\lesssim 10^{-7}$, i.e., $\lesssim 1$ per $(210 \mathrm{cMpc})^{3}$ volume. Using this cluster abundance, including BOSS1441, our survey volume of $10^{8} \mathrm{cMpc}$ should contain $\lesssim 10$ protoclusters with $M_{z=0} \gtrsim 10^{15} h^{-1} M_{\odot}$.

Chiang et al. (2014) found three overdensities at $z \approx 2.5$ with $\delta_{g}>10$ in the COSMOS field with a volume of $3.4 \times 10^{8} \mathrm{cMpc}^{3}$. Using this abundance, we expect that one overdensity with $\delta_{g}>10$ can be found in a $\approx 10^{8} \mathrm{cMpc}^{3}$ volume. Thus, BOSS1441 represents the most overdense region found from our initial survey with a $10^{8} \mathrm{Mpc}^{3}$ volume. In Table 4, we listed a sample of the most massive large-scale structures. All of these large-scale structures are spectroscopically confirmed and have overdensity measurements on large scales of 15-20 cMpc (Steidel et al. 1998; Venemans et al. 2002; Steidel et al. 2005; Venemans et al. 2005, 2007; Digby-North et al. 2010; Lee et al. 2014; Zheng et al. 2016). These large-scale structures we listed have galaxy overdensities of $\delta_{g}=2-6$ on $\approx 15-20 \mathrm{cMpc}$ scales. BOSS1441 has a comparable or higher galaxy overdensity compared to that of the previously confirmed protoclusters.

In our survey volume (see Section 2.4), we indeed find 11 fields that contain groups of strong IGM Ly $\alpha$ absorption. According to our cosmological simulation (Cai et al. 2016), the mass overdensities associated with the 11 absorption groups have a wide distribution, with the range of $\delta_{m}=0.6-2.8$. This wide range of mass overdensities arises from the intrinsic scatter between $\tau_{\text {eff }}$ and mass (see Figure 3 of Cai et al. 2016). If the 11 fields have a mass distribution similar to our simulations, then $30 \%$ of the 11 absorption ( $3-4)$ groups should have $\delta_{m}>2.2$ which corresponds to $10^{15} M_{\odot}$. Future observations on these 11 absorption groups will provide stringent constraint on the mass distribution provided by simulations.

\section{Summary and Future Prospects}

In this paper, we present an extremely massive overdensity BOSS1441 at $z=2.32 \pm 0.02$, discovered by utilizing our new approach of identifying overdensities from concentrations of strong IGM Ly $\alpha$ absorption systems (Cai et al. 2016). BOSS1441 is traced by a strong IGM Ly $\alpha$ absorption group with $\tau_{\text {eff }} \geqslant 3.0 \times\langle\tau\rangle$ within $15 h^{-1} \mathrm{cMpc}$. The absorption group is defined as $\geqslant 4$ absorption systems within the projected $20 h^{-1} \mathrm{cMpc}$ scale and at $z=2.32 \pm 0.02$ (Figures 2 and 3). BOSS1441 is also associated with multiple BOSS QSOs at similar redshift.

Our KPNO-4 m/MOSAIC narrowband and multiple-wavelength broadband observations have revealed a large-scale structure extending over $25 h^{-1} \mathrm{cMpc}$ scale. Using the two LBT/MODS masks, we have spectroscopically confirmed 19 LAEs (Figure 7 and Table 2). The LAE density is $11.8 \pm 2.8 \times$ that in random fields in a $(15 \mathrm{cMpc})^{3}$ volume $\left(\delta_{g}=10.8 \pm 2.8\right)$, making BOSS1441 one of the most overdense fields discovered to date. We estimate that BOSS1441 should collapse to a virialized cluster with $M_{z=0} \gtrsim 10^{15} M_{\odot}$. The number of such massive clusters should be $\sim 1$ within a $10^{7} \mathrm{cMpc}^{3}$ volume.

More observations are required to study the galaxy properties in such an overdense environment at $z=2.3$. We will use the Hubble Space Telescope Wide Field Camera 3 (WFC3) to conduct deep imaging in this field. These observations will test the existence of density-morphology relations at $z=2.3$. Also, we plan to enlarge our sample of large-scale structures at $z=2-3$ to fully constrain the cosmic structure formation and better characterize the environmental dependence of galaxy properties. In the near future, we aim to construct a uniform sample of the most massive overdensities at $z=2-3$ traced by strong intergalactic Ly $\alpha$ absorption systems. We have conducted a narrowband and broadband survey on other candidate fields by utilizing KPNO-4 m/MOSAIC and CFHT/MegaCAM. Future multiple-wavelength follow-ups and spectroscopic endeavors will quantify the overdensities, the diversity of galaxy populations, and the interactions between galaxy and IGM in the density peak at $z=2-3$.

We acknowledge the insightful comments from the anonymous referee. Z.C. acknowledges the helpful discussions with Yi-Kuan Chiang, Arjun Dey, and Catlin Casey. Z.C., X.F., and I.M. thank the support from US NSF grant AST 11-07682. Z.C. and J.X.P. acknowledge support from NSF AST-1412981. A.Z. acknowledges support from NSF grant AST-0908280 and NASA grant ADP-NNX10AD47G. N.K. acknowledges supports from JSPS grant 15H03645. Based on observations at Kitt Peak National Observatory, National Optical Astronomy Observatory (NOAO Prop. ID: 2013A-0434; PI: Z.C.; NOAO Prop. ID: 2014A-0395; PI: Z.C.), which is operated by the Association of Universities for Research in Astronomy (AURA) under cooperative agreement with the National Science Foundation. The authors are honored to be permitted to conduct astronomical research on Iolkam Du'ag (Kitt Peak), a mountain with particular significance to the Tohono O'odham. The LBT is an international collaboration among institutions in the United States, Italy, and Germany. The LBT Corporation partners are: The University of Arizona on behalf of the Arizona university system; Istituto Nazionale di Astrofisica, Italy; LBT Beteiligungsgesellschaft, Germany, representing the Max Planck Society, the Astrophysical Institute Potsdam, and Heidelberg University; The Ohio State University; The Research Corporation, on behalf of The University of Notre Dame, University of Minnesota, and University of Virginia. 


\section{References}

Adelberger, K. L., Shapley, A. E., Steidel, C. C., et al. 2005, ApJ, 629, 636 Adelberger, K. L., Steidel, C. C., Shapley, A. E., \& Pettini, M. 2003, ApJ, 584,45

Ahn, C. P., Alexandroff, R., Allende Prieto, C., et al. 2014, ApJS, 211, 17

Bahcall, N. A., \& Cen, R. 1993, ApJL, 407, L49

Bahcall, N. A., McKay, T. A., Annis, J., et al. 2003, ApJS, 148, 243

Becker, G. D., Hewett, P. C., Worseck, G., \& Prochaska, J. X. 2013, MNRAS, 430, 2067

Blakeslee, J. P., Franx, M., Postman, M., et al. 2003, ApJL, 596, L143

Bolton, A. S., Schlegel, D. J., Aubourg, É., et al. 2012, AJ, 144, 144

Bruzual, G., \& Charlot, S. 2003, MNRAS, 344, 1000

Byard, P. L., \& O'Brien, T. P. 2000, Proc. SPIE, 4008, 934

Cai, Z., Fan, X., Noterdaeme, P., et al. 2014, ApJ, 793, 139

Cai, Z., Fan, X., Peirani, S., et al. 2016, ApJ, 833, 135

Cai, Z., Fan, X., Yang, Y., et al. 2017, ApJ, 837, 71

Calzetti, D., Armus, L., Bohlin, R. C., et al. 2000, ApJ, 533, 682

Cantalupo, S., Arrigoni-Battaia, F., Prochaska, J. X., Hennawi, J. F., \& Madau, P. 2014, Natur, 506, 63

Casey, C. M. 2016, ApJ, 824, 36

Casey, C. M., Cooray, A., Capak, P., et al. 2015, ApJL, 808, L33

Chiang, Y.-K., Overzier, R., \& Gebhardt, K. 2013, ApJ, 779, 127

Chiang, Y.-K., Overzier, R., \& Gebhardt, K. 2014, ApJL, 782, L3

Ciardullo, R., Gronwall, C., Wolf, C., et al. 2012, ApJ, 744, 110

Dawson, K. S., Schlegel, D. J., Ahn, C. P., et al. 2013, AJ, 145, 10

De Lucia, G., Weinmann, S., Poggianti, B. M., Aragón-Salamanca, A., \& Zaritsky, D. 2012, MNRAS, 423, 127

Dey, A., Lee, K.-S., Reddy, N., et al. 2016, ApJ, 823, 11

Dickinson, M., Papovich, C., Ferguson, H. C., \& Budavári, T. 2003, ApJ, 587, 25

Digby-North, J. A., Nandra, K., Laird, E. S., et al. 2010, MNRAS, 407, 846

Drory, N., Salvato, M., Gabasch, A., et al. 2005, ApJL, 619, L131

Elbaz, D., Daddi, E., Le Borgne, D., et al. 2007, A\&A, 468, 33

Fassbender, R., Nastasi, A., Böhringer, H., et al. 2011, A\&A, 527, L10

Feldmann, R., \& Mayer, L. 2015, MNRAS, 446, 1939

Ford, A. B., Oppenheimer, B. D., Davé, R., et al. 2013, MNRAS, 432, 89

Gawiser, E., Francke, H., Lai, K., et al. 2007, ApJ, 671, 278

Giallongo, E., Ragazzoni, R., Grazian, A., et al. 2008, A\&A, 482, 349

Gobat, R., Daddi, E., Onodera, M., et al. 2011, A\&A, 526, A133

Guaita, L., Acquaviva, V., Padilla, N., et al. 2011, ApJ, 733, 114

Gunn, J. E., Siegmund, W. A., Mannery, E. J., et al. 2006, AJ, 131, 2332

Hennawi, J. F., Prochaska, J. X., Cantalupo, S., \& Arrigoni-Battaia, F. 2015 , Sci, 348, 779
Hu, E. M., McMahon, R. G., \& Egami, E. 1996, ApJL, 459, L53

Jenkins, A., Frenk, C. S., White, S. D. M., et al. 2001, MNRAS, 321, 372

Kollmeier, J. A., Weinberg, D. H., Davé, R., \& Katz, N. 2003, ApJ, 594, 75

Lee, K.-G., Bailey, S., Bartsch, L. E., et al. 2013, AJ, 145, 69

Lee, K.-S., Dey, A., Hong, S., et al. 2014, ApJ, 796, 126

Lee, K.-G., Hennawi, J. F., White, M., Croft, R. A. C., \& Ozbek, M. 2014, ApJ, 788, 49

Lee, K.-G., Suzuki, N., \& Spergel, D. N. 2012, AJ, 143, 51

Martin, D. C., Matuszewski, M., Morrissey, P., et al. 2015, Natur, 524, 192

Mo, H. J., \& White, S. D. M. 1996, MNRAS, 282, 347

Muldrew, S. I., Hatch, N. A., \& Cooke, E. A. 2015, MNRAS, 452, 2528

Noterdaeme, P., Petitjean, P., Carithers, W. C., et al. 2012, A\&A, 547, LL1

Oppenheimer, B. D., Davé, R., Katz, N., Kollmeier, J. A., \& Weinberg, D. H. 2012, MNRAS, 420, 829

Pâris, I., Petitjean, P., Aubourg, É, et al. 2012, A\&A, 548, A66

Peter, A. H. G., Shapley, A. E., Law, D. R., et al. 2007, ApJ, 668, 23

Postman, M., Franx, M., Cross, N. J. G., et al. 2005, ApJ, 623, 721

Prescott, M. K. M., Dey, A., \& Jannuzi, B. T. 2009, ApJ, 702, 554

Reddy, N. A., Steidel, C. C., Pettini, M., et al. 2008, ApJS, 175, 48

Reed, D., Gardner, J., Quinn, T., et al. 2003, MNRAS, 346, 565

Ross, N. P., Myers, A. D., Sheldon, E. S., et al. 2012, ApJS, 199, 3

Sawyer, D. G., Daly, P. N., Howell, S. B., Hunten, M. R., \& Schweiker, H. 2010, Proc. SPIE, 7735, 77353A

Smith, G. P., Kneib, J.-P., Smail, I., et al. 2005, MNRAS, 359, 417

Springel, V. 2005, MNRAS, 364, 1105

Stark, C. W., White, M., Lee, K.-G., \& Hennawi, J. F. 2015, MNRAS, 453,311

Steidel, C. C., Adelberger, K. L., Dickinson, M., et al. 1998, ApJ, 492, 428

Steidel, C. C., Adelberger, K. L., Shapley, A. E., et al. 2005, ApJ, 626, 44

Uchimoto, Y. K., Yamada, T., Kajisawa, M., et al. 2012, ApJ, 750, 116

van Dokkum, P. G., Whitaker, K. E., Brammer, G., et al. 2010, ApJ, 709, 1018

Venemans, B. P., Kurk, J. D., Miley, G. K., et al. 2002, ApJL, 569, L11

Venemans, B. P., Röttgering, H. J. A., Miley, G. K., et al. 2005, A\&A, 431, 793

Venemans, B. P., Röttgering, H. J. A., Miley, G. K., et al. 2007, A\&A, 461,823

Vogelsberger, M., Genel, S., Springel, V., et al. 2014, MNRAS, 444, 1518

Wang, T., Elbaz, D., Daddi, E., et al. 2016, arXiv:1604.07404

White, M., Myers, A. D., Ross, N. P., et al. 2012, MNRAS, 424, 933

Yang, Y., Zabludoff, A., Tremonti, C., Eisenstein, D., \& Davé, R. 2009, ApJ, 693, 1579

Zheng, Z.-Y., Malhotra, S., Rhoads, J. E., et al. 2016, ApJS, 226, 23 